\title{
Robotics from the Investor's Point of View: How to Profit from the Rise of Robots
}

\author{
Andrea Forni, CFTe \\ InvestiRobot-Observatory on Investment in Robotics, FRN Trading Strategies, Milano 20125, Italy
}

\begin{abstract}
This research paper evaluates whether Robotics equities traded on Stock Markets worldwide are a good investment opportunity or not. Robotics seems to become more and more attractive for both institutional investors (who act rationally and prefer medium to long term investments) and individual investors (who are driven by irrational behaviour and prefer short term investments). In order to forecast if robotics is an investment opportunity that could inflate a new financial bubble in the oncoming years, this research paper compares robotics equities with Internet equities and analyses what happened during the Dot-com Mania in the 1990s. A number of similarities come out as well as several key enabling factors that confirm robotics is a major investment opportunity for the next decade.
\end{abstract}

Key words: Robotics stocks, financial bubbles, investor behaviour, key investment factors, risk management, stock market.

\section{Introduction}

This research paper wants to answer a very simple question: "Is robotics attractive for investors today?" In order to answer the question, for the sake of simplicity we divided the investors into two main categories based on their investment behavior: the professional investors and the individual investors (the general public). The former behave quickly and rationally and prefer medium to long-term investments. They search for companies that have solid fundamentals including product lines, market leadership, good management and stable finances. Mizrahi [1] reported that quality companies do not trade at attractive prices for very long. For this reason, Professional Investors move fast and buy into the capital of quality companies at the starting phases of the Capital Market lifecycle.

According to Dr. Jean-Paul Rodrigue of Hofstra University [2] Capital Markets (and bubbles) go through stages. Fig. 1 shows a generic Capital Market lifecycle model which is applicable to any financial

Corresponding author: Andrea Forni, director of the Observatory on Investment in Robotics at FRN Trading Strategies, research field: development of robotics ecosystem models applied to the financial analysis of robotics companies. products like a single equity or a stock market index. In the first two phases of the capital market lifecycle (namely "Smart Money” and “Institutional Investors”) Professional Investors can buy shares at a good valuation which reflects the assets of the company: technology, management and products. If robotics is interesting for investors, institutional ones should already own shares of robotics companies, today!

\section{Ownership}

This is the starting point of this research paper and the first key factor we investigated to confirm our initial assumption. We studied the ownership of well-known robotics companies in order to find who their main investors are. Fig. 2 shows a partial view of the whole outcome of our research. Finding most of the big names in Finance like BlackRock, T.Rowe Price and J.P. Morgan to name the few is no surprise. They have been pouring a lot of money into robotics over the last years. This is a good sign to confirm our assumption.

\section{Private and Public Initiatives}

Sometimes money and Big Finance are not enough to launch a new disruptive and pervasive technology 


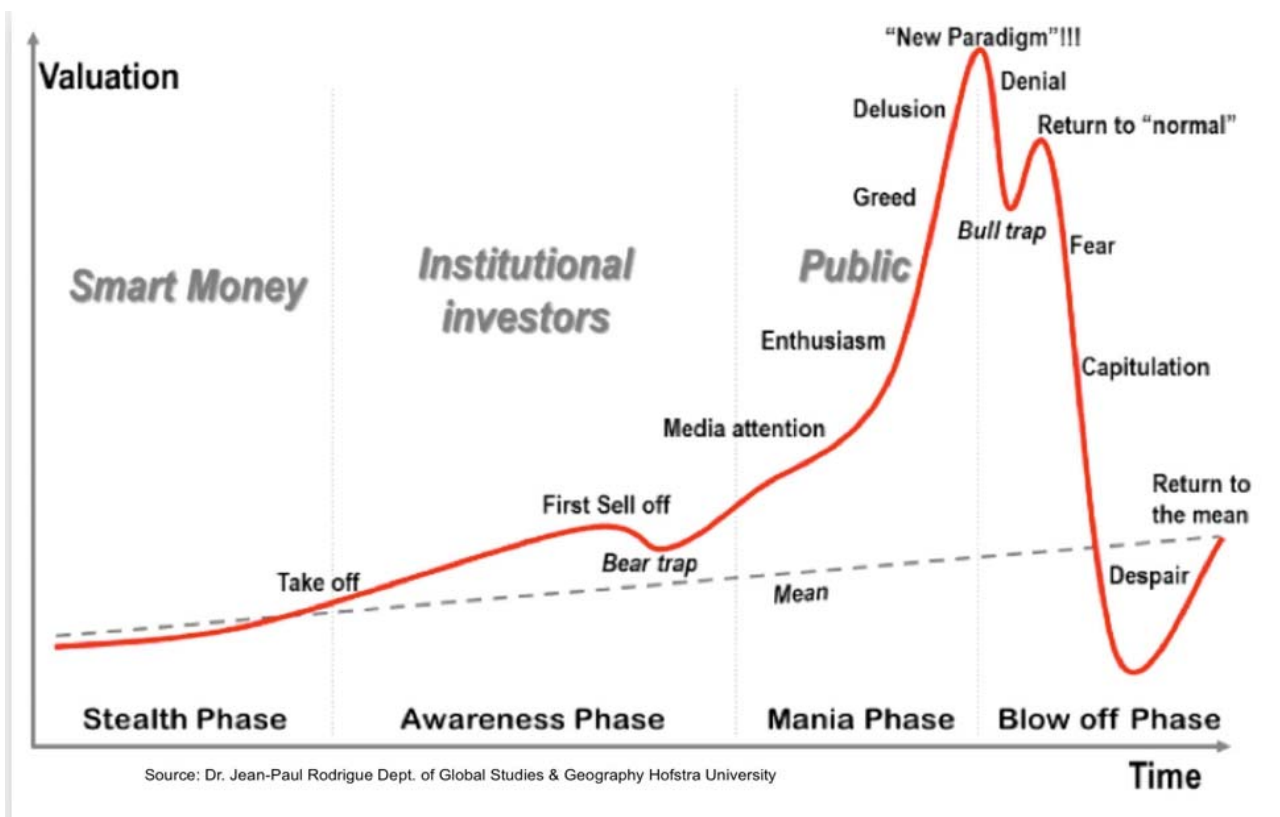

Fig. 1 Generic Capital Market Lifecycle Model.

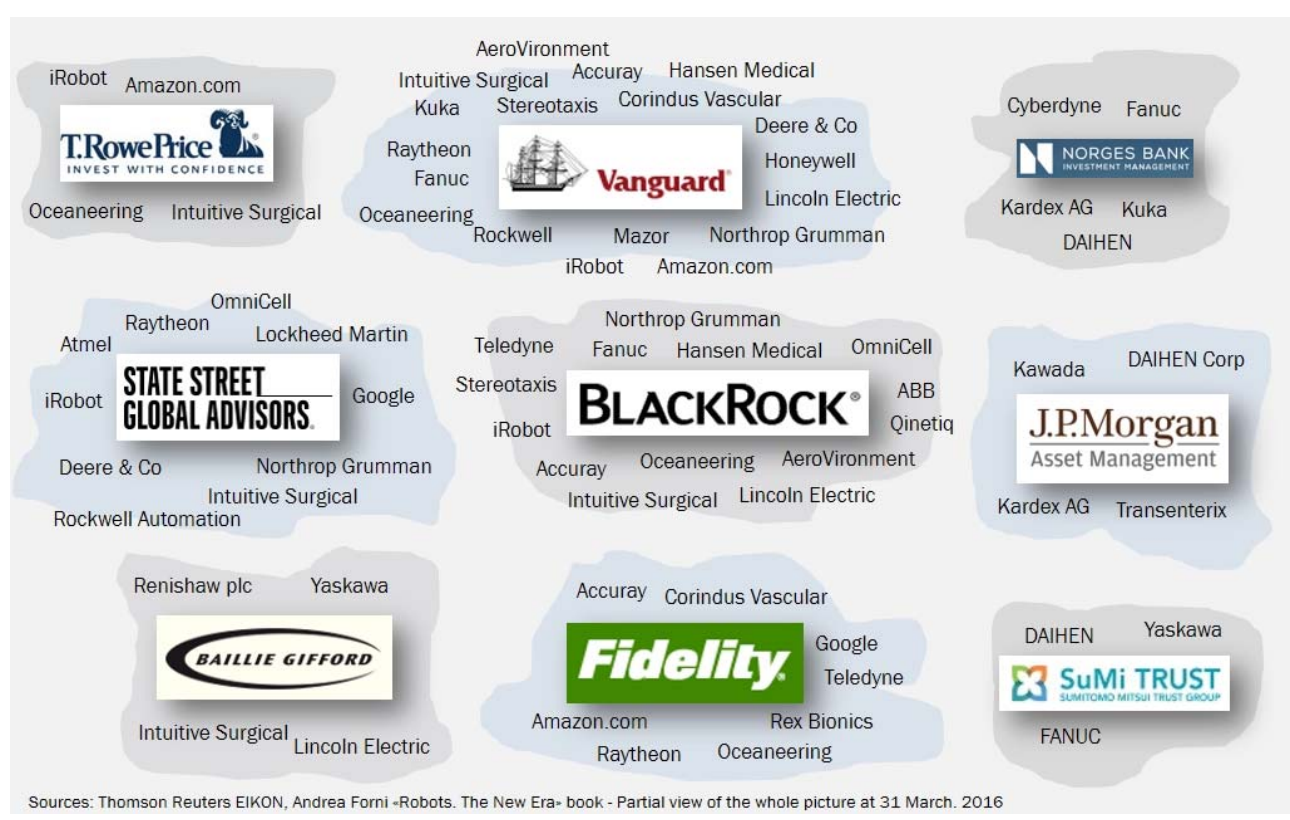

Fig. 2 Ownership of robotics companies (partial view of the whole picture).

like robotics. We decided to investigate who are the big players in robotics R\&D\&I. This is a second key factor to confirm our assumption and it is represented in Fig. 3. We found innumerable initiatives by public and private entities that are contributing to forming a technological and cultural critical mass that will facilitate the introduction of robotics.

This is a very partial view of the whole set of the initiatives we have found worldwide, but it is enough to give the flavour. What is really important is the fact that Robotics is attracting both public entities and very powerful and visionary players like Google, Amazon, Apple, Facebook. They have financial power, technology power, lobbying power and influential power to create the market for any robotics product they would like to launch in the next future. 


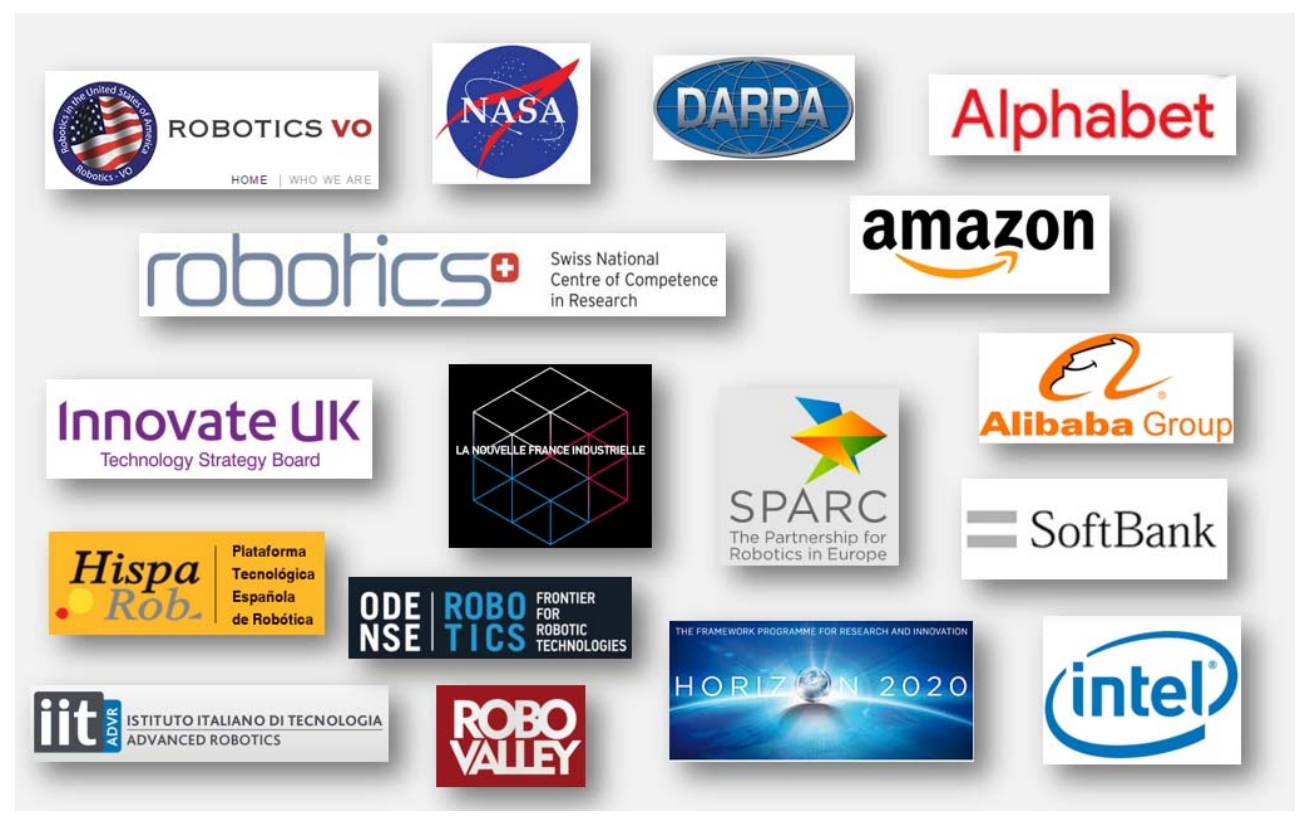

Fig. 3 Big players enabling the development of robotics (partial view of the whole picture).

\section{Incumbents Disrupt Traditional Industries and Influence Legislators to Act}

The disruptive effect that these visionary and powerful Internet players are bringing to more traditional sectors can be understood by looking at a research made by Appinions.com [3] about who are the 10 biggest influencers in the development of the autonomous car. This research highlighted that the first two influencers in the ranking list are not traditional car makers (Google and Intel), as well as Apple in 7th place and Rio Tinto in 10th place.

The entrance of these big and powerful players into the old Automotive industry is good because they can really bring it to new life. But investors must ask themselves if traditional car makers will keep the pace with these new players or will succumb in the next 15 to 20 years. Answering this question is very important before any investment decision in the Automotive Industry or in the ownership of any single car maker.

The entrance of players like Google and Apple in the robotics race is also good because they have the power to influence legislators to act in favour of a special interest. Several researches have pointed out that the mass introduction of Service Robotics cannot be possible without new regulation in many sectors: present-day the first examples come from the aviation authorities and the transportation authorities that are already working on this matter.

\section{Robotics Market is Fast-Growing}

The third key factor that we usually study investigating a new fast-growing technology is the market for its products. As we have already pointed out, in the long run the valuation of the company (and its stock price) is correlated to earnings growth and sales growth. Market data and forecasts produced annually by the IFR Statistical Department and published in their World Robotics Report [4] confirm the Robotics market is constantly expanding both in Units sold and in Value for the years to come.

Industrial Robotics Markets totalled 229,261 units sold in $2014(+29 \% \mathrm{Y} / \mathrm{Y})$ for a total market value of USD 10.7 billion $(+13 \% \mathrm{Y} / \mathrm{Y})$. And the forecast the period years 2015-2018 are in favour of a rise in units sold $(1,300,000+15 \% \mathrm{Y} / \mathrm{Y})$ and in market value (USD 60.4 Billion). Same astonishing numbers for the Service Robotics Market (Professional Use): total units sold in 2014: 24,207 units, $+11 \% \mathrm{Y} / \mathrm{Y}$; total market value: $\$ 3.77$ billion, $+3 \%$. The forecast is also very 
attracting with 152,375 units sold and a Market Value of USD 19.6 Billion over the period years 2015-2018. These data and forecast are a very good sign for long term investors like the institutional ones.

\section{Wide Selection of Robotics Equities}

The fourth key factor we investigated in our research is the availability of a consistent number of robotics equities to choose from. From a behavioural point of view, investors behave like children in an ice cream parlour: the more flavours they can choose, the more likely they will find something suitable to their risk profile and their portfolios. As shown in Fig. 4, robotics allows to invest in a wide choice of equities traded in the Stock Markets worldwide.

We have created a proprietary database that contains 160 public companies dealing with robotics. Among them (at the date of writing this paper), 27 companies can be classified as pure-play robotics businesses which are fully dedicated to develop, manufacture and sell robotics products; 92 of them are companies which have diversified their product lines by including robotics in their vertical or horizontal offer; the last 41 companies are classified as ancillary to the robotics businesses providing technologies, products and engineering services. In their whole, despite the fact that these 160 public companies are widespread among 31 GICS (Global Industry Classification Standard) sub-sectors it is worth to notice a strong concentration of these equities in just three sectors, namely “Aerospace \& Defense”, “Healthcare Equipment” and "Industrial Machinery". The same results come from the analysis of the geographical diversification.

Despite these 160 companies are headquartered across 15 countries we have noticed their concentration in just a bunch of trading stock markets like (in order of weight): USA, Japan, Israel, UK and Germany. On the other hand, we have found a good diversification in Market Capitalization and Sizing that enables investors to choose among Micro, Small, Medium and Big Caps.

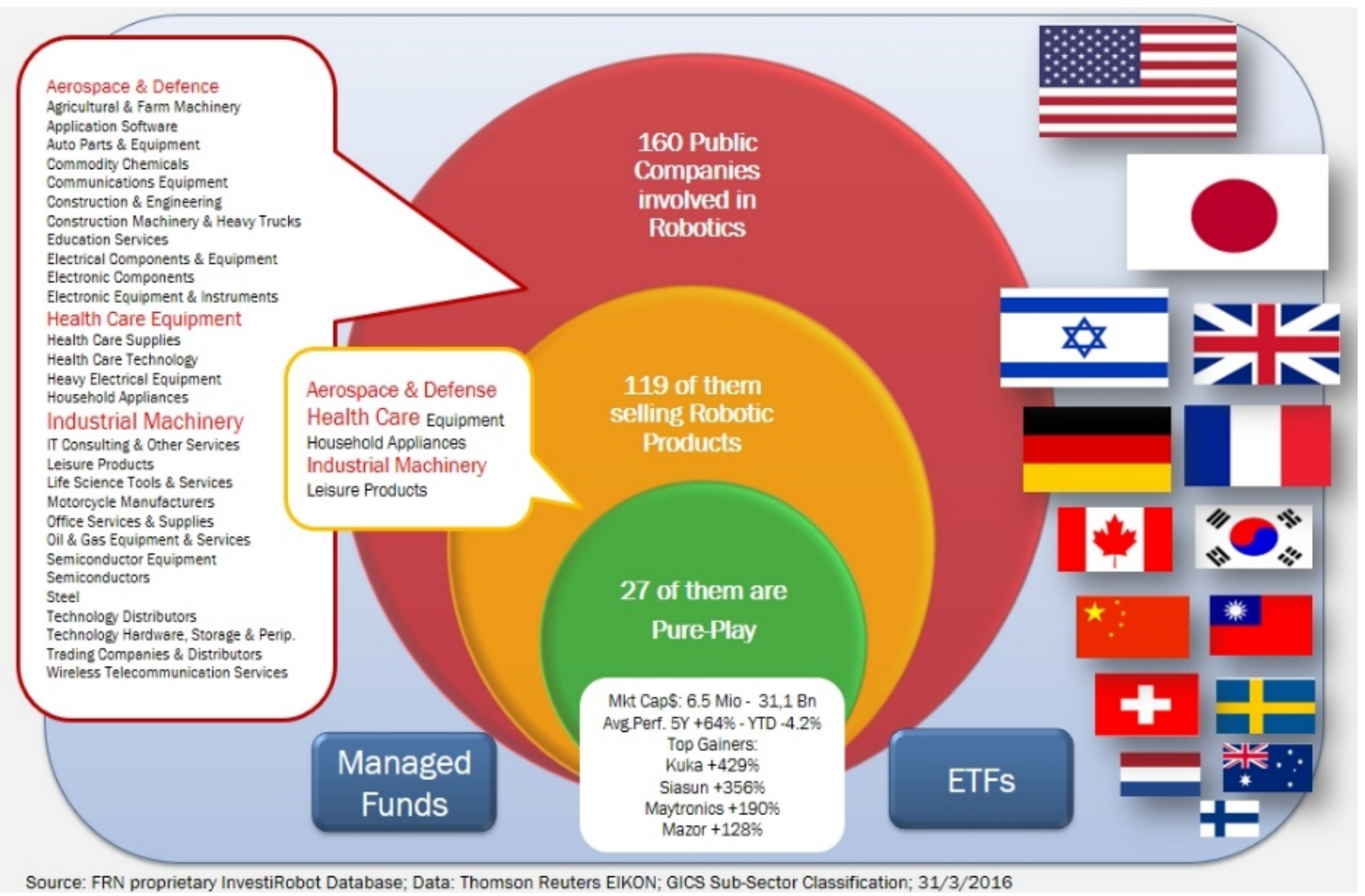

Fig. 4 Public companies listed on major Stock Exchanges and their distribution worldwide. 
Price performances as well as fundamental valuations are also highly diversified among different sectors and among companies belonging to the same sector.

Apart from the huge number of equities already available it's also interesting to highlight the fact that some benchmark indexes and investment vehicles like EFTs and managed Funds are appearing on the market enabling investors to more easily invest in robotics.

\section{A View from the Past: The Rise of the Internet Industry and the Dot-com Bubble}

An important part of our research involved the study of what happened in the past when a new disruptive technology took place. Many of us can clearly remember the rise of the Internet Industry twenty years ago and the irrational behaviour triggered by the bubble inflation at the end of the 1990s. This phenomenon has been well documented and we are plenty of resources to study. Fig. 5 reported by Kampas Research [5] shows the price action of the NASDAQ Index from 1990 to 2005 and highlights which key factors were critical for the expansion of the Internet market and the rise of expectations which inflated the dot-com bubble.

By comparing Fig. 5 with Fig. 1 it is possible to clearly identify how the bubble inflated over the four stages of the Capital Market Lifecycle and the sequence of events that enabled the emergence of the Internet as disruptive technology. Firstly, the entrance of venture capitals in this new technological sector in the early 1990s; secondly, the emergence of the Internet in the mid-1990s; thirdly, the entrance of new aggressive players like Amazon and Google, followed by an increasing number of IPOs (Initial Public Offering) of Internet stocks; finally, the boost to the market was given by the Internet Tax Freedom Act signed into law on October 21 by President Bill Clinton; last but not least, on February 1999 the launch of the Dow Jones Internet Index composed by 40 public companies officially marked the birth of the new Internet Industry on the financial market.

\section{Risk Associated to Technology Investment}

After reviewing what happened twenty years ago, we also remind our readers that financial investors must always care about potential risks. Let just highlight some of them. Technology is the driver for the acceptance by the consumer market. Think of what happens in the smartphones market every new announcement of some manufacturer. People are rushing to buy the new models more easy-of use, with better batteries and with

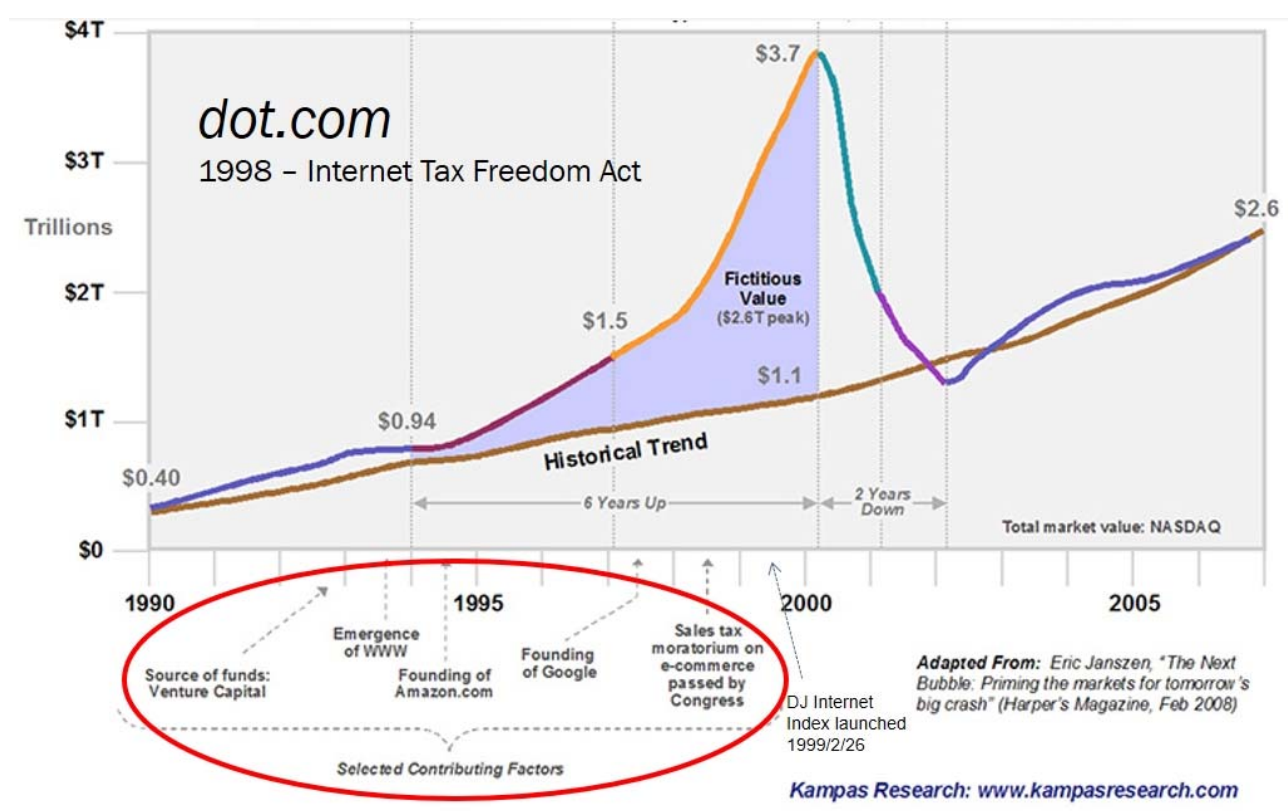

Fig. 5 The Capital Market Lifecycle Model applied to the NASDAQ Index from 1990 to 2005. 
astonishing design. If a technology company is not able to constantly innovate and keep the pace with the competitors the value of that company will fall down as a consequence. Investing in robotics stocks is like betting on the ability of the company to stay on top the robotics race in the next years. Other risks, like those associated to the use or misuse of robots, can be covered with portfolio risk management techniques. What could happen if an autonomous car is attacked by hackers and crashes killing all people on board? The stock price of that specific car maker will sell-off immediately.

Some other risks could not be forecasted like social risks, occupational risks, and ethical risks because they are more related to human behavior. The inclusion of Service Robots in new workplaces will lead to a redefinition of the labor market. What will happen when millions of fast-food workers, or delivery boy will feel threatened by the automation of their jobs decided by the management of their companies? Think of millions of people going on strike, or acting against the new technology with sabotages. What is the impact on the Profit \& Loss of these companies? And how is it reflected by their stocks?

\section{News Media, Investment Behaviour and Financial Bubbles}

Now, we will quickly explore the universe of the individual investors. We are moving from the rational world of the institutional investors to the irrational world of the Individual Investors who act driven by emotions. As Charles Mizrahi [1] reported in his book, they buy equities as they buy lottery tickets attracted by sexy stories and the illusion of a great payoff.

Back to our capital market life cycle shown in Fig. 1, the general public enters the market during the so-called Mania Phase. This phase is ignited by the Media attention. The News Media are fundamental propagators of speculative price movements through their efforts to make news interesting to their audience. The entrance of a huge number of irrational buyers in a financial market where there is scarcity of equities lifts the stock prices to unsustainable levels in a very short time, giving the price chart the typical exponential shape. And the bubble starts inflating. The stock price does not represent anymore the valuation of the business. It represents the expectations of the individual investors to become very rich.

Given the fact that the Mania Phase is ignited by media attention, Robotics is the perfect technology to help journalist write sexy stories and exciting headlines for their articles. Robotics products are cool, nice-looking and easy to be understood by the general public. We are not surprised to see a number of important magazines showing robotics at their cover pages like recently we found in Scientific American, Time, Wired, the Economist just to name the few. This is another important key factor in our research. In order to understand the role of the Media Industry in inflating the general public expectations we studied what happened during the Dot-com Mania. The result of a research was made by the BBC in United Kingdom [7] on the use of the phrase "dot-com" in UK newspapers between 1997 and 2000. The phrase "dot-com" appeared in just six articles in UK newspapers on February 1999 and then sky-rocketed to an amazing number of 427 articles a year later on February 2000. The dramatic increase of these articles from August 1999 on and the exponential shape of the curve of the graph would have to warn everyone that a financial bubble was about to burst! For the years to come, it's worth also to remind that the irrational behavior of individual investors flocking into the financial market in search of juicy opportunities in robotics will favor stocks easily recognizable. In order to support our view, we can remind the events of years 1996-2000 when people scrambled to acquire any stocks with the name containing ".com" or "web" or "internet". The results of a research made by Liu and Song [8] on a sample of 95 small companies that changed their names to include ".com" or "internet" demonstrate that these stocks had an average abnormal return of $89 \%$ for the 
10 days surrounding the announcement of the name change. If the investors are more inclined to short-term speculation they should also base their selection criteria on a psychological analysis of the behavior of the general public in order to select those stocks that will be more attractive in an irrational market.

\section{Funding Robotics Start-ups: Initial Public Offering vs. Venture Capital}

Another key factor we investigated is the increasing number of IPOs and Venture Capital funding over the years and their correlation with both the development of the market for a new disruptive technology and the rise of expectations from the general public. As Fig. 6 shows, until now in robotics we have witnessed an increasing number of funding at a reasonable growth rate while IPOs are still lacking. Nothing compared to what happened during the Dot-com Mania Phase when we witnessed a sky-rocketing number of new IPOs in the final stage of the bubble between years 1996 and 2000 .

\section{Conclusions}

Let us draw some conclusions. We have seen pros and cons of investing in Robotics. The answer of the initial question "Is Robotics attractive as an investment opportunity?” is quite positive. Robotics is attractive for the long run since this industry in its whole has all the qualities the professional investors look for: a solid business model; visionary entrepreneur leadership; ability to fulfil present and future market needs, in a fast growing market; solid fundamentals. Given these facts, the proper stock-picking of robotics stocks is still very difficult and time-consuming, since a "Robotics" sector classification is still missing and robotics companies are still classified by the output category of their products. It is also quite difficult to identify robotics companies by searching only for their names since few of them have "robot" or "robotics" in their name present day.

What about a new bubble in the oncoming years? To reassure the reader, let us draw a distinction between the dot-com companies of the 1990s and the robotics companies of today. This time the product is real, tangible and already successfully applied in many economic sectors. We are not confronted by simple business ideas passed off as companies with high growth potential through a new evaluation model invented by investment banks! Or at least, not yet. Robotic applications have been used in manufacturing

Tech IPO - Dot.com Mania 1996-2002

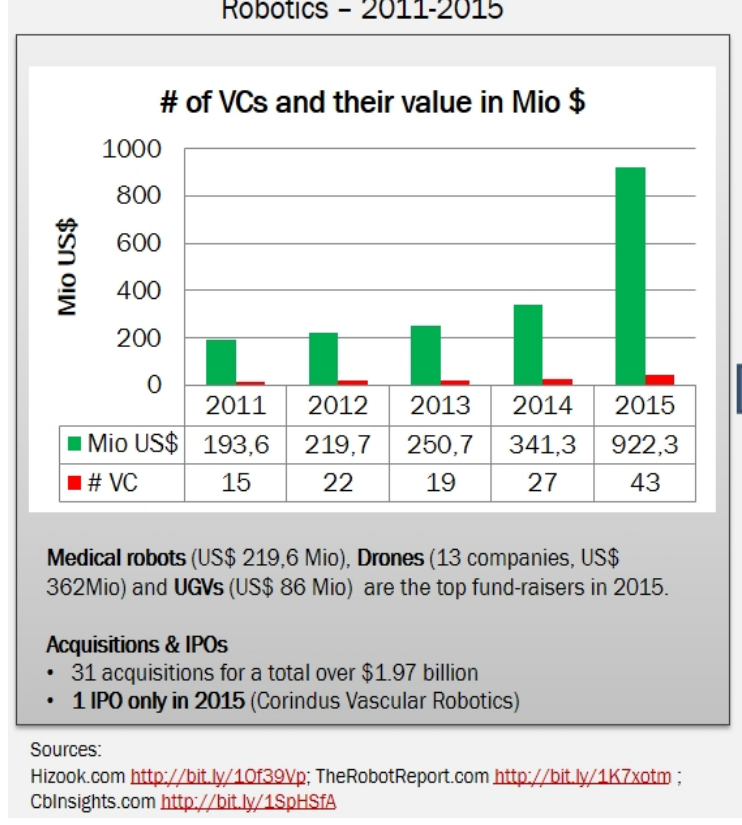

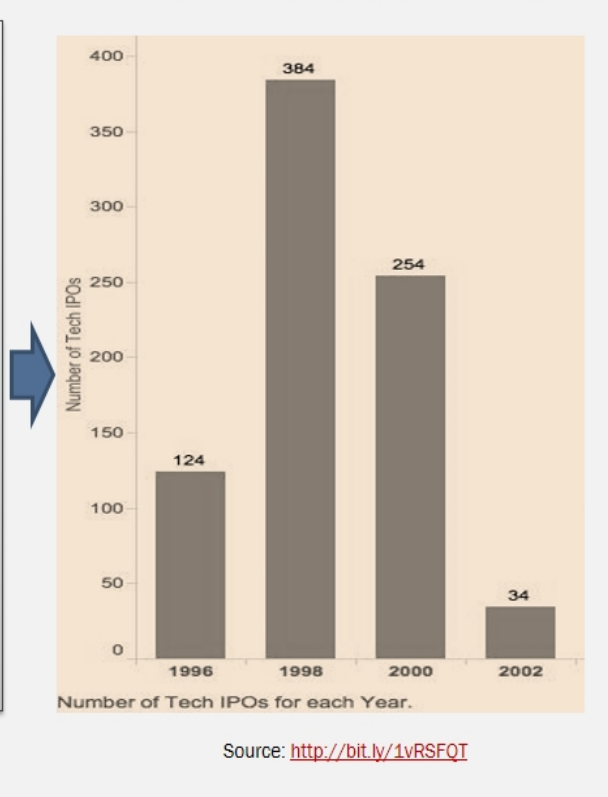

Fig. 6 Comparing Robotics and the Dot-com Era: IPOs vs. VC funding. 
industry for decades and have recently been adopted (in other forms) in spheres such as the hospital, aerospace and subsea exploration sectors as well as, obviously, for military purposes. Research programmes are being financed by governments, major industrial groups and leading universities. Start-up companies developing an innovation quickly find funding and are acquired for millions of dollars by major groups such as Google which seems to have a huge appetite for robotics.

Even if we are not in favor of a new bubble on Robotics, we think that we should experience some volatility when the crowd will discover Robotics as a sexy investment for the short run. And even if a new bubble will inflate, the experience of year 2000 shows that although many of the Internet companies were wiped out when the bubble burst, those with the best fundamentals survived and have prospered up to the present day, handsomely rewarding those investors who took the long-term view.

\section{References}

[1] Mizhrai, C. 2008. Getting Started in Value Investing. United States of America: John Wiley \& Sons.

[2] Rodrigue, J. Phases of a bubble. As reported in Keenan, M. 2011. A Bubble under Microscope, Sunday Times, June 26.

[3] Appinions.com. 2014. Autonomous Cars: An Industry Study, case study on website www.appinions.com.

[4] IFR Statistical Department. 2015. World Robotics Report 2015, International Federation of Robotics, Germany.

[5] Janszen, E. 2008. The Next Bubble, Harper's Magazine, adapted by Kampas Research and reported in: Kampas Research website 2008. Mapping the Rise and fall of Market Bubbles, blog post.

[6] Bhattacharya, U., Galpin, N., Ray, R., and Yu, X. 2006. "The Role of the Media in the Internet IPO Bubble." Journal of Financial and Quantitative Analysis 44 (3): 657-82.

[7] BBC. 2000. Dot.com: Just a bit too dot.common? BBC News e-cyclopedia.

[8] Liu, Q., and Song. F. 2001. "The Rise and Fall of Internet Stocks: Should Financial Analysts Be Blamed?” SSRN Electronic Journal, March 2001. 